\title{
Modeling ultrafast all-optical switching in synthetic ferrimagnets
}

\author{
S. Gerlach,,${ }^{1, *}$ L. Oroszlany, ${ }^{2}$ D. Hinzke, ${ }^{1}$ S. Sievering, ${ }^{1}$ S. Wienholdt, ${ }^{1}$ L. Szunyogh, ${ }^{3}$ and U. Nowak ${ }^{1}$ \\ ${ }^{1}$ Fachbereich Physik, Universität Konstanz, D-78457 Konstanz, Germany \\ ${ }^{2}$ Department of Physics of Complex Systems, Eötvös University, Pázmány Péter sétány 1/A, H-1117 Budapest, Hungary \\ ${ }^{3}$ MTA-BME Condensed Matter Research Group and Department of Theoretical Physics, \\ Budapest University of Technology and Economics, Budafoki út 8, HU-1111 Budapest, Hungary
}

(Received 7 March 2017; revised manuscript received 24 May 2017; published 28 June 2017)

\begin{abstract}
Based on numerical simulations, we demonstrate thermally induced magnetic switching in synthetic ferrimagnets composed of multilayers of rare-earth and transition metals. Our findings show that deterministic magnetization reversal occurs above a certain threshold temperature if the ratio of transition-metal atoms to rare-earth atoms is sufficiently large. Surprisingly, the total thickness of the multilayer system has little effect on the occurrence of switching. We further provide a simple argument to explain the temperature dependence of the reversal process.
\end{abstract}

DOI: 10.1103/PhysRevB.95.224435

\section{INTRODUCTION}

The demonstration of helicity-dependent all-optical magnetization switching [1,2] was one of the most surprising findings in ultrafast magnetization dynamics. The experiments showed that magnetization switching is possible solely triggered by a single laser pulse in the subpicosecond range avoiding any externally applied magnetic field. These experiments were performed on $\mathrm{GdFeCo}$, a rare-earth-based ferrimagnet where the rare-earth (RE) sublattice is antiferromagnetically coupled to the transition-metal (TM) sublattice. First attempts to describe these unexpected processes were based on the assumption that the circularly polarized laser pulse induces a strong magnetic field via the inverse Faraday effect, which determines the direction of the switching [2,3]. Overall, this process takes place on a time scale that is orders of magnitude shorter than today's writing procedures in hard discs. This calls for applications in magnetic data storage, and alternative materials including alloys [4], heterostructures [5], and synthetic ferrimagnets [6] are currently being investigated.

The discovery of thermally induced all-optical switching using linearly polarized light [7,8] cast a new light on alloptical switching and called for more sophisticated models since this switching works without any external or optically induced magnetic field, which could define the magnetization direction during its recovery after the ultrafast quenching.

In simulations, this switching was observed in an atomistic spin model developed by Ostler et al. [7-9]. An attempt to explain the occurrence of the transient ferromagneticlike state (TFMLS) was given by Mentink et al., who identified angular momentum transfer driven by the intersublattice exchange as the crucial process [10], which was also investigated in more detail by Bar'yakhtar et al. [11]. Later on, the thermally induced switching was more quantitatively described by means of an orbital-resolved spin model, where the magnetic moments stemming from $d$ electrons of the TM, $d$ electrons of the RE, and $f$ electrons of the RE are distinguished [12]. Here, it was shown that the initial laser excitation brings the sublattices into a strong nonequilibrium after $1 \mathrm{ps}$. This

\footnotetext{
*stefan.gerlach@uni-konstanz.de
}

happens due to the different demagnetization times of the individual sublattices [13]. On that time scale, electron and phonon temperatures are nearly equilibrated below $T_{\mathrm{C}}$ again, but the Fe sublattice is already completely demagnetized while the Gd sublattice remains still rather ordered. The remagnetization dynamics of Fe taking place subsequently leads to a state where the Gd spins and the $\mathrm{Fe}$ spins are aligned - the transient ferromagneticlike state. The TFMLS arises naturally as a consequence of a redistribution of the energy and angular momentum between the different sublattices due to the maximization of entropy under the constraint of energy and angular momentum conservation. These processes, which are driven via the precession term of the Landau-Lifshitz-Gilbert equation, dominate on shorter times scales. The following relaxation back to a ferrimagnetic equilibrium state is on a longer time scale, where dissipative processes are responsible, and does not necessarily lead to a switched state $[4,14]$. The details of this relaxation process depend on the material properties as well as the experimental specifications and are still under investigation.

In the following, we will explore the possibility of thermally induced switching in synthetic ferrimagnets comprised of bilayers of $\mathrm{Fe}$ and $\mathrm{Gd}$. We use $a b$ initio methods to estimate spin model parameters for Fe-Gd bilayers. The dynamic simulations of the spin model allow for an investigation of the preconditions for thermally induced switching. We find that deterministic magnetization reversal occurs only above a certain threshold temperature and in bilayers where the ratio of transition-metal atoms to rare-earth atoms is sufficiently large. Finally, we find a simple explanation as to why the compensation temperature is so important.

\section{MODEL}

Our aim is to model a synthetic ferrimagnet as a bilayer of two ferromagnets, Fe and Gd, with a negative coupling between the two layers. For that, we consider an atomistic spin model where localized spins are arranged on a simplecubic lattice structure. Spins experience effective exchange interactions with their nearest neighbors and dipole-dipole interaction is neglected since we are only interested in the short-time magnetization dynamics, which is governed by 
exchange interactions. The Heisenberg Hamiltonian of the system studied reads

$$
\mathcal{H}=-\sum_{\mathrm{NN}} J_{i j} \mathbf{S}_{i} \mathbf{S}_{j}-\sum_{i} d_{z} S_{i, z}^{2}
$$

The first term represents the Heisenberg exchange energy, where the exchange interaction is either between spins of the Fe layer, spins of the Gd layer, or across the interface between $\mathrm{Fe}$ and Gd spins. This term contains, therefore, three interactions, $J_{\mathrm{Fe}-\mathrm{Fe}}, J_{\mathrm{Gd}-\mathrm{Gd}}$, and $J_{\mathrm{Fe}-\mathrm{Gd}}$. The second term represents a uniaxial anisotropy with anisotropy constant $d_{z}$. The lateral dimensions of the model are $150 \times 150$ atoms with the layers stacked along the $z$ axis and with periodic boundary conditions in transverse directions. The thicknesses of the $\mathrm{Fe}$ and Gd layers are varied.

$A b$ initio calculations have been performed in terms of the fully relativistic screened Korringa-Kohn-Rostoker (KKR) method, designed, in particular, for layered systems and surfaces [15]. The local spin-density approximation (LSDA) parametrization from Ref. [16] was used. The strong correlation of the localized $4 f$ states of the Gd atoms was treated within the framework of the LSDA $+U$ approach [17] as implemented within the KKR method [18]. The calculations were carried out with the commonly used $U=6.7 \mathrm{eV}$ and $J=0.7 \mathrm{eV}$ values of the Coulomb and exchange integrals [17] and the double-counting term derived in Refs. [19,20] satisfying the atomic limit for the LSDA total energy. The exchange constants have been obtained by means of the relativistic torque method [21].

The geometry used in the $a b$ initio calculations were based on a heterostructure of six Fe layers between two semi-infinite bulk Gd regions. For the hcp structure of Gd bulk, we used the experimental $c / a$ ratio of 1.5904 and an optimized lattice constant $a=3.450 \AA$ [22]. The interlayer distance in the Fe region was chosen such that the volume per $\mathrm{Fe}$ atom was identical to that in bcc Fe with the experimental lattice constant of $2.867 \AA$. The distance of the Fe planes to the nearest Gd planes was taken to be the average of the Fe-Fe and Gd-Gd layer distances. The interlayer exchange interactions obtained by the $a b$ initio procedure were then mapped to the simple cubic structure with effective nearest-neighbor interactions used in the spin-dynamics simulations.

The calculations outlined above result in exchange constants of the spin model above with the ratios $J_{\mathrm{Fe}-\mathrm{Fe}}: J_{\mathrm{Gd}-\mathrm{Gd}}$ : $J_{\mathrm{Fe}-\mathrm{Gd}}=1: 0.286:-0.388$, which we use in the subsequent dynamic simulations. The magnetic moments of $\mathrm{Fe}$ and Gd have the values of $\mu_{\mathrm{TM}}=1.92 \mu_{\mathrm{B}}$ and $\mu_{\mathrm{RE}}=7.63 \mu_{\mathrm{B}}$, referring to the values found for bulk FeGd in Ref. [12]. These values are close to those obtained in our KKR LSDA $+U$ calculations. In addition, we use an anisotropy constant of $d_{z}=0.2 \mathrm{meV}$ favoring magnetization along the $z$ axis.

The dynamics of the system is governed by the stochastic Landau-Lifshitz-Gilbert equation of motion,

$$
\frac{\left(1+\alpha^{2}\right) \mu_{i}}{\gamma} \dot{\mathbf{S}}_{i}=-\mathbf{S}_{i} \times\left[\mathbf{H}_{i}+\alpha\left(\mathbf{S}_{i} \times \mathbf{H}_{i}\right)\right],
$$

with the gyromagnetic ratio $\gamma$ and a dimensionless Gilbert damping constant $\alpha$ that describes the coupling to the heat bath. In our simulations, the damping constant is set to $\alpha=0.02$ $[12,23]$. Thermal fluctuations are included as an additional

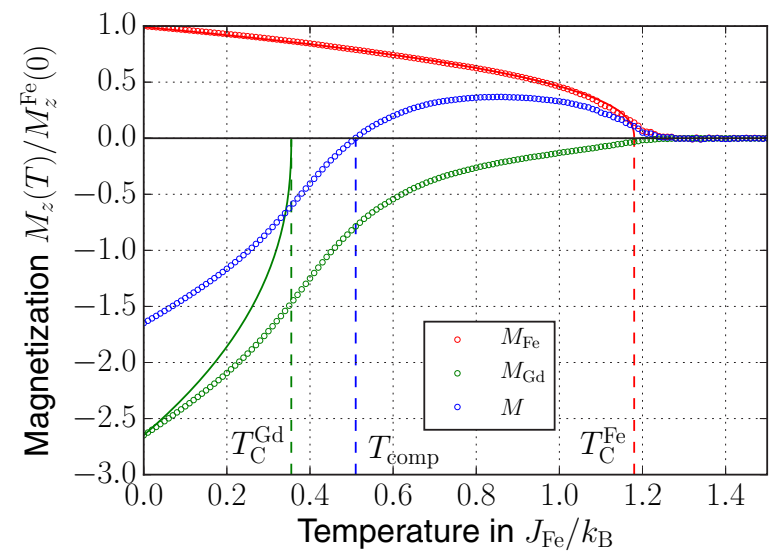

FIG. 1. Temperature-dependent equilibrium magnetization for a bilayer with three monolayers of Fe and two monolayers of Gd. The magnetization of the element with the stronger intralayer exchange interaction, in this case $\mathrm{Fe}$, closely follows the shape of a ferromagnet with its Curie temperature $T_{\mathrm{C}}^{\mathrm{Fe}}$ close to the Curie temperature of the coupled system. The element with the weaker intralayer exchange interaction, in this case $\mathrm{Gd}$, exhibits magnetic ordering above its own bulk Curie temperature, $T_{\mathrm{C}}^{\mathrm{Gd}}$, due to the interaction with the other sublattice. The chosen ratio of layer thicknesses leads to a larger magnetic moment of the Gd layer at low temperatures and, consequently, to a magnetic compensation point at a temperature $T_{\text {comp }}$ which is slightly above the bulk Curie temperature of the $\mathrm{Gd}$.

white-noise term $\zeta_{i}$ in the internal fields $\mathbf{H}_{i}=-\frac{\partial \mathcal{H}}{\partial \mathbf{S}_{i}}+\zeta_{i}(t)$, with

$$
\left\langle\zeta_{i}(t)\right\rangle=0, \quad\left\langle\zeta_{i \eta}(0) \zeta_{j \theta}(t)\right\rangle=\frac{2 k_{\mathrm{B}} T \alpha \mu_{i}}{\gamma} \delta_{i j} \delta_{\eta \theta} \delta(t),
$$

where $i, j$ denote lattice sites and $\eta, \theta$ are Cartesian components. All of the algorithms we use are described in detail in Ref. [24].

In Fig. 1, we present the equilibrium properties of a bilayer consisting of three monolayers of $\mathrm{Fe}$ and two monolayers of Gd. The equilibrium magnetization, calculated as the spatial and time average of the magnetic moments over the simulated multispin system and given time interval, shows a typical temperature-dependent decrease due to increasing spin fluctuations. This bilayer system behaves as a synthetic ferrimagnet with a magnetic compensation point $T_{\text {comp }}$ at a temperature of about $40 \%$ of the Curie temperature $T_{\mathrm{C}}$.

It is well known that laser-induced demagnetization in transition metals is several times faster than in rare-earth elements $[25,26]$. Several approaches have been proposed to explain this behavior, including electron-phonon scattering processes of the Elliott-Yafet type [27] and intra-atomic energy transfer within the electronic subsystem [12]. However, based on the different demagnetization time scales, we may assume that heating a multilayer system with incident laser light can lead to a situation where the TM layer is completely demagnetized, while the RE layers still retain a substantial net magnetization. We use this fact in the following and do not calculate the action of the laser pulse on the spin system explicitly. Instead we focus on the relaxation of the magnetization at constant temperature, starting our simulations with a spin configuration where the Fe sublattice is completely 
demagnetized (spins are randomly oriented) while we vary the degree of magnetization of the Gd layer. This initial Gd magnetization and the temperature will turn out to be crucial quantities for the understanding of thermally triggered switching.

\section{RESULTS}

For a fixed layer configuration, we treat the initial RE magnetization remaining after the laser excitation and the temperature $T$ as the relevant parameters which determine the magnetization dynamics triggered by the laser pulse. In Fig. 2, three different possible scenarios are shown, switching (top), switching followed by switching back to the initial state (center), and no switching (bottom). The chosen values for $T$ and $\mathrm{Gd}$ magnetization are indicated in Fig. 3. The switching scenario corresponds to the work of Radu et al. [7], while the backswitching was measured by Khorsand et al. [28]. Note that in all three cases, the magnetization of the transition metal starts towards negative values (while the original sign before demagnetization by the effect of the laser heating would have been positive) so that a TFMLS is obtained. Note also that the transverse components of the magnetization are usually not small-apart from the case of switching-which indicates a linear mechanism for the case of successful switching but a more precessional process for the case of no switching and backswitching.

A systematic variation of the two parameters, i.e., temperature and initial Gd magnetization, allows for the construction of a switching diagram, as shown in Fig. 3. It is ternary in the way that it provides information about the relaxation process, with the three scenarios above (switching, backswitching, or no switching), as shown in Fig. 2. The diagram is constructed by taking into account the magnetization dynamics of the two species involved for a single run during a time interval of $40 \mathrm{ps}$. We identify three distinct regions: a connected no-switching region (red), a connected switching region (cyan-blue), and a backswitching region along the boundary of the other two regions (yellow-orange).

For an interpretation of this diagram, we first note that for very low values of initial RE magnetization, one would expect the system to randomly pick one of the two possible equilibrium configurations, i.e., either switched or not. This sort of statistical behavior is indeed evident from the isolated data points at the left-hand side of the diagram. Furthermore, one would expect that high values of $M_{\mathrm{RE}}$ would prevent the system from switching at low temperatures because the level of order in the RE layer is too high to become demagnetized. This is indeed what we find from Fig. 3. The no-switching region is found in the bottom-right corner, corresponding to low temperature and high $M_{\mathrm{RE}}$.

So far, the switching diagram meets our intuitive expectations. It is less obvious, however, why high temperatures (above $T_{\text {comp }}$ ) lead to switching regardless of how strongly the RE layers are demagnetized by the heat pulse. Qualitatively, this can be understood keeping in mind the linear switching mechanism, which avoids transverse magnetization components (see Fig. 2 and Refs. [2,29]). Linear switching needs a high degree of spin disorder in the system and, consequently elevated temperatures.
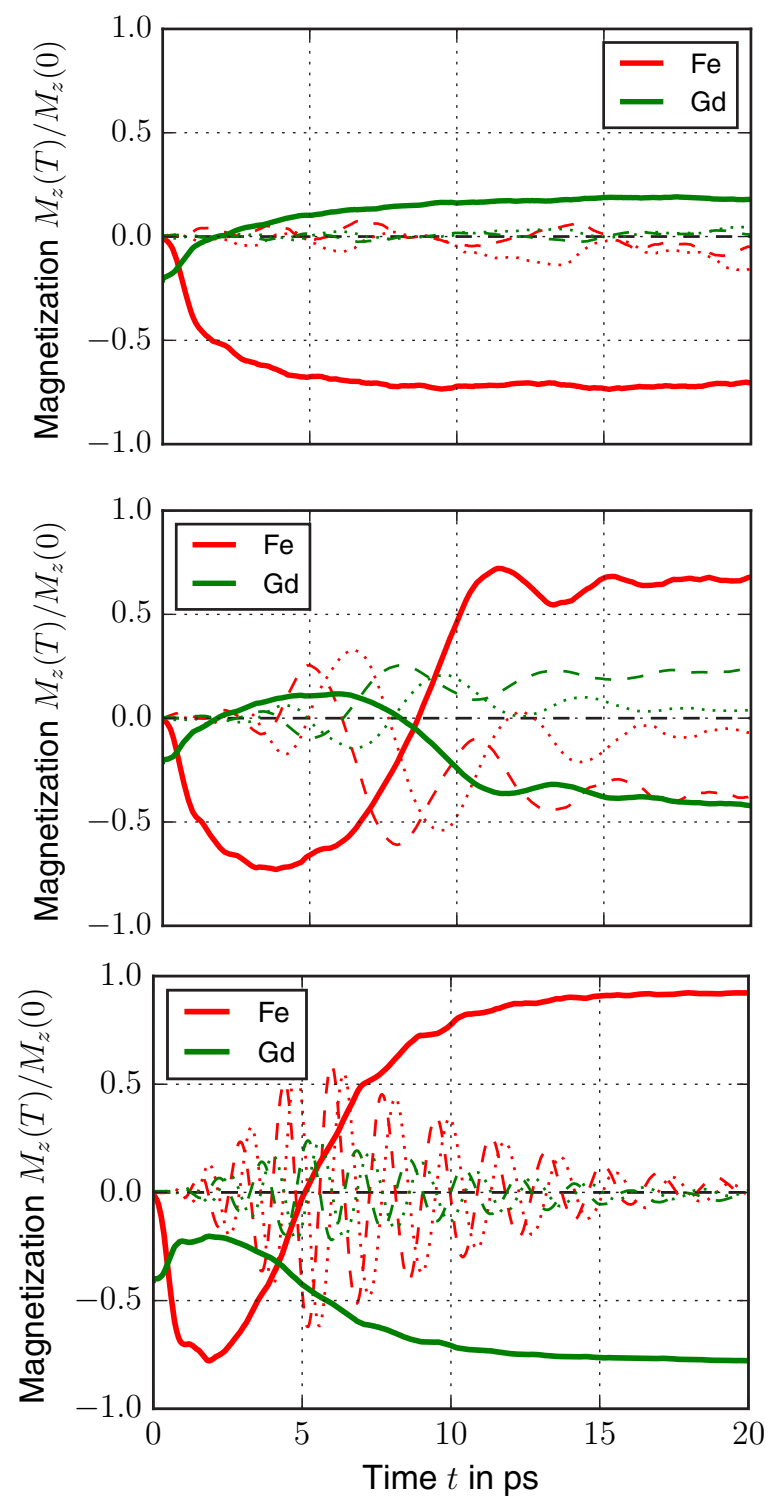

FIG. 2. The three possible time-relaxation scenarios for the sublattice magnetizations of a RE-TM layered system. The chosen values for $\mathrm{T}$ and $\mathrm{Gd}$ magnetization are indicated in Fig. 3. Top: Magnetization switches with respect to the initial configuration. Center: Both sublattice magnetizations change their signs twice, ending up back in the initial state. Bottom: No switching, i.e., the rare-earth layer magnetization does not change sign. Also shown are the transverse components (dotted and dashed lines). For the case of backswitching and no switching, these components are of the order of the magnitude of the magnetization.

More quantitatively, the role of temperature can be understood via the equilibrium layer magnetizations, as shown in Fig. 1, since those mark the final values for the relaxation process. Let us assume that following the excitation of the laser pulse, the Fe layer is completely demagnetized while the $\mathrm{Gd}$ is only demagnetized by $50 \%$. The bilayer is far from equilibrium and a relaxation process will set in of which the details depend on the temperature. Each layer will relax towards its individual, temperature-dependent equilibrium value of magnetization. 


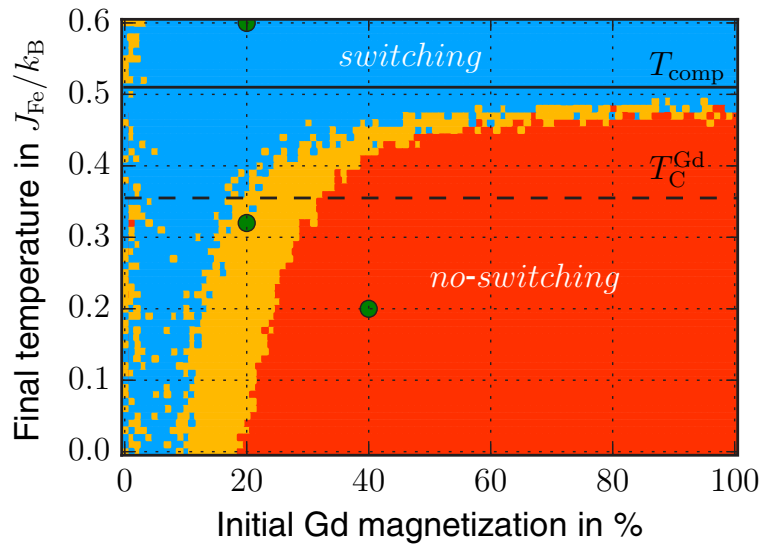

FIG. 3. Switching in a 3:2 layer sample after 40 ps. Color coding: Magnetization switches (cyan-blue), magnetization does not switch and returns to the initial state (red), and both magnetizations change their sign twice, ending up in the initial state (backswitching) (yelloworange). The compensation temperature $T_{\text {comp }}$ is indicated by a solid black line; the Curie temperature $T_{\mathrm{C}}^{\mathrm{Gd}}$ of the isolated Gd layer is indicated by a dashed black line. The three points indicate the chosen values for the scenarios in Fig. 2.

We can identify three important temperature ranges:

(i) $T<T_{\mathrm{C}}^{\mathrm{RE}}$ : Both layers tend to increase their net magnetization magnitudes (absolute value) towards higher values.

(ii) $T_{\mathrm{C}}^{\mathrm{RE}}<T<T_{\mathrm{C}}^{\mathrm{TM}}$ : To reach equilibrium, the magnitude of the TM magnetization still increases, while the magnitude of the RE magnetization tends to decrease.

(iii) $T>T_{\mathrm{C}}^{\mathrm{TM}}$ : The system will completely demagnetize, i.e., the magnetization of both layers vanishes.

For most TM-RE layer ratios, $T_{\text {comp (if it exists) is higher }}$ than $T_{\mathrm{C}}^{\mathrm{RE}}$ and only the temperature range between $T_{\mathrm{C}}^{\mathrm{RE}}$ and $T_{\mathrm{C}}^{\mathrm{TM}}$ supports the dynamics necessary for deterministic switching-decreasing Gd magnetization and increasing $\mathrm{Fe}$ magnetization. Consequently, the temperature must be at least above the critical temperature of bulk Gd to show deterministic switching. Below $T_{\mathrm{C}}^{\mathrm{RE}}$ only nondeterministic switching could be observed, i.e., the switching is not reproducible. We checked this by repeating the simulations ten times for a certain range of parameter above and below $T_{\mathrm{C}}^{\mathrm{RE}}$ while changing the random numbers to generate different thermal fluctuations. Above the compensation temperature, switching is always possible. That means if $T_{\text {comp }}$ is very low, switching can also be done below $T_{\mathrm{C}}^{\mathrm{RE}}$.

The next question is why the Fe layer magnetization starts recovering towards negative magnetization which results in a TFMLS. This is a consequence of angular momentum conservation, as was already pointed out by several authors $[10,12]$. While the Gd still demagnetizes (since the temperature is above the bulk critical temperature of $\mathrm{Gd}$ ), the dynamics of the Fe layer magnetization must change into the other direction keeping the angular momentum constant. The argument also explains that one needs a certain initial Gd magnetization to start with. Without initial Gd magnetization, there is no angular momentum reservoir to drag the $\mathrm{Fe}$ magnetization towards negative values.

Angular momentum conservation is not strictly fulfilled in the spin system. The relaxation part of the equation of motion
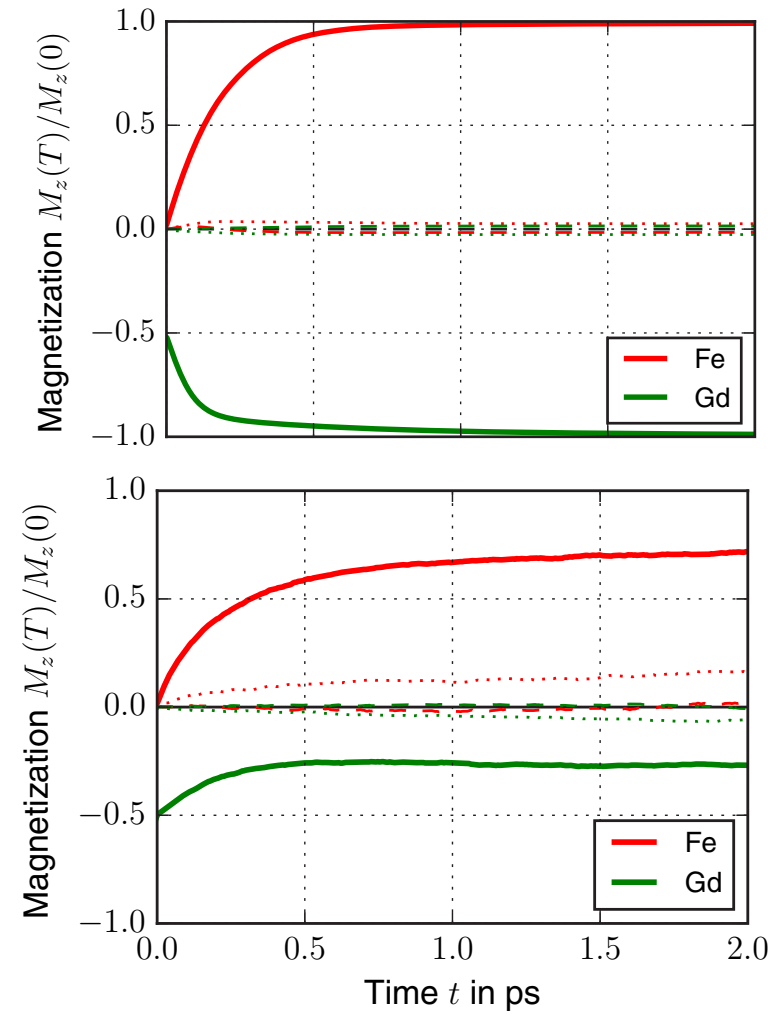

FIG. 4. Strong dissipation ( $\alpha=1$ ) in a 3:2 layer sample, implying no conservation of angular momentum. The relaxation does not lead to a TFMLS either at low temperature where the Gd magnetization increases or at high temperature where it decreases. Top: $T<T_{\mathrm{C}}^{\mathrm{Gd}}$. Bottom: $T_{\mathrm{C}}^{\mathrm{Gd}}<T<T_{\mathrm{C}}^{\mathrm{Fe}}$. Also shown are the transverse components (dotted and dashed lines) which remain small for both cases.

breaks this conservation on time scales which are determined by the value of the damping constant $\alpha$. For low values of $\alpha$, the precessional part of the equation of motion is much larger, leading to dynamics which keeps total energy and total angular momentum conserved on shorter-time scales. This is different when considering larger values of $\alpha$. For comparison, we investigate in Fig. 4 the regime of strong dissipation $(\alpha=1)$. Here, the time scales of precessional dynamics are of the same order as the time scale of relaxation, and dissipative effects counteract the conservation of angular momentum in the system. Only if the damping constant $\alpha$ is sufficiently small, angular momentum is almost conserved on short-time scales, along with the total energy, leading to a TFMLS as seen in Fig. 2. The figure also illustrates that-depending on the temperature - the Gd magnetization might relax either towards higher or lower equilibrium values. The transition from dissipationless dynamics to the regime where damping effects dominate the dynamics has previously been investigated [12].

In the following, we turn to the peculiarities of the layered system. The layer-resolved magnetization in Fig. 5 shows the importance of the interface layers for switching. For low damping, the angular momentum conservation leads to a relaxation dynamics with an exchange of angular momentum between the still demagnetizing Gd layer and the Fe layer, leading to a negative Fe magnetization and, consequently, to 


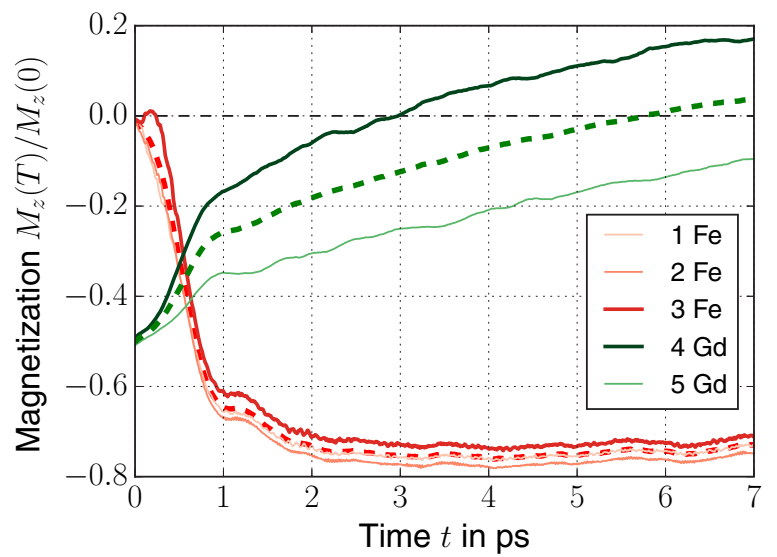

FIG. 5. Layer-resolved magnetization dynamics for switching in a 3:2 layer sample. The Gd layer is demagnetized to $50 \%$ of its zero-temperature value. The bold lines correspond to the layers at the $\mathrm{Gd}-\mathrm{Fe}$ interface and the dashed lines show the average value for $\mathrm{Gd}$ or Fe. The temperature is $T=0.5 J_{\mathrm{Fe}} / k_{\mathrm{B}}$.

a TFMLS. Because of the antiferromagnetic coupling along the $\mathrm{Fe}$-Gd interface, the $\mathrm{Fe}$ interface monolayer lags behind the other layers. After some ps, however, the Fe magnetization has reached its new, negative equilibrium value, pushing the Gd via the negative interface coupling towards positive values. Here, the dynamics is quicker at the interface as for the other Gd layer which is lagging behind.

We also simulated other layer thicknesses and ratios. While the Fe-Gd bilayer with $20-40 \%$ Gd (for example 4:1, 3:1, or 3:2 layer) turned out to have the correct thickness ratio for switching (and having a magnetization compensation temperature), we found that the overall thickness is less relevant. Successful switching can also be seen in much bigger samples (although on longer-time scales), for instance in a 30:20 Fe-Gd layer. The antiferromagnetic coupling of the interface layers finally leads to a switching of all layers when the temperature of the heat bath exceeds $T_{\text {comp }}$ (which is almost the same as $T_{\mathrm{C}}^{\mathrm{Gd}}$ for big samples). Changing the ratio of the $\mathrm{Fe}$ and $\mathrm{Gd}$ layer does not change the switching behavior as long as the sample maintains a magnetization compensation temperature. Only the temperature range for switching increases with decreasing percentage of Gd.

\section{SUMMARY}

We explored thermally induced magnetic switching in synthetic ferrimagnets composed of a bilayer of rare earth and transition metal on the basis of spin model simulations where the model parameters were calculated from first principles. Varying the temperature and the degree of initial rare-earth magnetization directly after the laser pulse, one may find either backswitching, or no switching. Deterministic magnetization reversal occurs above a certain threshold temperature which is above the bulk Curie temperature of the rare earth since only then the magnetization of the rare-earth sublattice relaxes towards lower magnitude. The optimal ratio of transition-metal atoms to rare-earth atoms for successful switching has 20-40\% Gd layer, while the total thickness of the multilayer system only affects the time scale of switching.

\section{ACKNOWLEDGMENTS}

This work has been funded by the Center for Applied Photonics at the University of Konstanz and by the Hungarian National Scientific Research Fund (NKFIH) under Projects No. K115575 and No. K108676. O.L. acknowledges support from the Janos Bolyai Scholarship of the Hungarian Academy of Sciences.
[1] C. D. Stanciu, F. Hansteen, A. V. Kimel, A. Kirilyuk, A. Tsukamoto, A. Itoh, and T. Rasing, Phys. Rev. Lett. 99, 047601 (2007).

[2] K. Vahaplar, A. M. Kalashnikova, A. V. Kimel, D. Hinzke, U. Nowak, R. Chantrell, A. Tsukamoto, A. Itoh, A. Kirilyuk, and T. Rasing, Phys. Rev. Lett. 103, 117201 (2009).

[3] K. Vahaplar, A. M. Kalashnikova, A. V. Kimel, S. Gerlach, D. Hinzke, U. Nowak, R. Chantrell, A. Tsukamoto, A. Itoh, A. Kirilyuk, and T. Rasing, Phys. Rev. B 85, 104402 (2012).

[4] S. Alebrand, U. Bierbrauer, M. Hehn, M. Gottwald, O. Schmitt, D. Steil, E. E. Fullerton, S. Mangin, M. Cinchetti, and M. Aeschlimann, Phys. Rev. B 89, 144404 (2014).

[5] S. Mangin, M. Gottwald, C.-H. Lambert, D. Steil, V. Uhlî̃, L. Pang, M. Hehn, S. Alebrand, M. Cinchetti, G. Malinowski, Y. Fainman, M. Aeschlimann, and E. E. Fullerton, Nat. Mater. 13, 286 (2014).

[6] Y. Tsema, M. Savoini, A. Kirilyuk, A. Tsukamoto, and T. Rasing, in Ultrafast Magnetism I, Springer Proceedings in Physics, Vol 159, edited by J.-Y. Bigot, W. Hübner, T. Rasing, and R. Chantrell (Springer, Cham, 2015), p. 69.
[7] I. Radu, K. Vahaplar, C. Stamm, T. Kachel, N. Pontius, H. A. Durr, T. A. Ostler, J. Barker, R. F. L. Evans, R. W. Chantrell, A. Tsukamoto, A. Itoh, A. Kirilyuk, T. Rasing, and A. V. Kimel, Nature (London) 472, 205 (2011).

[8] T. A. Ostler, J. Barker, R. F. L. Evans, R. W. Chantrell, U. Atxitia, O. Chubykalo-Fesenko, S. E. Moussaoui, L. L. Guyader, E. Mengotti, L. J. Heyderman, F. Nolting, A. Tsukamoto, A. Itoh, D. Afanasiev, B. A. Ivanov, A. M. Kalashnikova, K. Vahaplar, J. Mentink, A. Kirilyuk, T. Rasing, and A. V. Kimel, Nat. Commun. 3, 666 (2012).

[9] T. A. Ostler, R. F. L. Evans, R. W. Chantrell, U. Atxitia, O. Chubykalo-Fesenko, I. Radu, R. Abrudan, F. Radu, A. Tsukamoto, A. Itoh, A. Kirilyuk, T. Rasing, and A. V. Kimel, Phys. Rev. B 84, 024407 (2011).

[10] J. H. Mentink, J. Hellsvik, D. V. Afanasiev, B. A. Ivanov, A. Kirilyuk, A. V. Kimel, O. Eriksson, M. I. Katsnelson, and T. Rasing, Phys. Rev. Lett. 108, 057202 (2012).

[11] V. G. Bar'yakhtar, V. I. Butrim, and B. A. Ivanov, JETP Lett. 98, 289 (2013).

[12] S. Wienholdt, D. Hinzke, K. Carva, P. M. Oppeneer, and U. Nowak, Phys. Rev. B 88, 020406 (2013). 
[13] B. Frietsch, J. Bowlan, R. Carley, M. Teichmann, S. Wienholdt, D. Hinzke, U. Nowak, K. Carva, P. M. Oppeneer, and M. Weinelt, Nat. Commun. 6, 8262 (2015).

[14] A. R. Khorsand, M. Savoini, A. Kirilyuk, A. V. Kimel, A. Tsukamoto, A. Itoh, and T. Rasing, Phys. Rev. Lett. 108, 127205 (2012).

[15] J. Zabloudil, R. Hammerling, P. Weinberger, and L. Szunyogh, Electron Scattering in Solid Matter, A Theoretical and Computational Treatise (Springer, Berlin, 2005).

[16] J. P. Perdew and A. Zunger, Phys. Rev. B 23, 5048 (1981).

[17] V. I. Anisimov, F. Aryasetiawan, and A. I. Lichtenstein, J. Phys.: Condens. Matter 9, 767 (1997).

[18] H. Ebert, A. Perlov, and S. Mankovsky, Solid State Commun. 127, 443 (2003).

[19] V. I. Anisimov, I. V. Solovyev, M. A. Korotin, M. T. Czyzyk, and G. A. Sawatzky, Phys. Rev. B 48, 16929 (1993).

[20] I. V. Solovyev, P. H. Dederichs, and V. I. Anisimov, Phys. Rev. B 50, 16861 (1994).

[21] L. Udvardi, L. Szunyogh, K. Palotás, and P. Weinberger, Phys. Rev. B 68, 104436 (2003).
[22] L. Oroszlány, A. Deák, E. Simon, S. Khmelevskyi, and L. Szunyogh, Phys. Rev. Lett. 115, 096402 (2015).

[23] F. Schlickeiser, U. Atxitia, S. Wienholdt, D. Hinzke, O. Chubykalo-Fesenko, and U. Nowak, Phys. Rev. B 86, 214416 (2012).

[24] U. Nowak, Handbook of Magnetism and Advanced Magnetic Materials, Vol. 2, Micromagnetism, edited by H. Kronmüller and S. Parkin (Wiley, Chichester, 2007).

[25] A. Vaterlaus, T. Beutler, and F. Meier, Phys. Rev. Lett. 67, 3314 (1991).

[26] E. Beaurepaire, J.-C. Merle, A. Daunois, and J. Y. Bigot, Phys. Rev. Lett. 76, 4250 (1996).

[27] B. Koopmans, G. Malinowski, F. D. Longa, D. Steiauf, M. Fahnle, T. Roth, M. Cinchetti, and M. Aeschlimann, Nat. Mater. 9, 259 (2010).

[28] A. R. Khorsand, M. Savoini, A. Kirilyuk, A. V. Kimel, A. Tsukamoto, A. Itoh, and T. Rasing, Phys. Rev. Lett. 110, 107205 (2013).

[29] N. Kazantseva, D. Hinzke, R. W. Chantrell, and U. Nowak, Europhys. Lett. 86, 27006 (2009). 